\title{
Asteroseismic observations of OB stars
}

\section{Peter De Cat $^{1}$, Katrien Uytterhoeven ${ }^{2}$, Juan Gutiérrez-Soto ${ }^{3}$, Pieter Degroote $^{4}$, and Sergio Simón-Díaz ${ }^{5}$}

\author{
${ }^{1}$ Royal observatory of Belgium, Ringlaan 3, B-1180 Brussel, Belgium \\ email: Peter.DeCat@oma.be \\ ${ }^{2}$ Lab. AIM, CEA/DSM-CNRS-Université Paris Diderot, CEA, IRFU, SAp, Saclay, 91191, \\ Gif-sur-Yvette, France \\ email: katrien.uytterhoeven@cea.fr \\ ${ }^{3}$ Instituto de Astrofísica de Andalucía (CSIC), Apartado 3004, 18080 Granada, Spain \\ email: jgs@iaa.es \\ ${ }^{4}$ Instituut voor Sterrenkunde, KU Leuven, Celestijnenlaan 200D, B-3001 Leuven, Belgium \\ email: Pieter.Degroote@ster.kuleuven. be \\ ${ }^{5}$ Instituto de Astrofísica de Canarias, E-38200 La Laguna, Tenerife, Spain \\ email: ssimon@iac.es
}

\begin{abstract}
The region of the hot end of the main-sequence is hosting pulsating stars of different types and flavours. Pulsations are not only observed for Slowly pulsating B stars (mid to late B-type stars; high order g-modes) and $\beta$ Cephei stars (early B-type stars; low order $\mathrm{p} / \mathrm{g}$-modes) but are also causing variability in Be stars and OB-supergiants. In this review we give an overview of the asteroseismic observations that are currently available for these types of stars. The first asteroseismic results were solely based on ground-based observations. Recently, the arrival of space-based data gathered by space missions like MOST, COROT and KEPLER has led to important discoveries for massive stars, highlighting their excellent asteroseismic potential. We show that, despite the unprecedented precision of the space-based data, there is still a clear need for ground-based follow-up observations.
\end{abstract}

Keywords. stars: early-type, stars: emission-line, Be, stars: variables: other, stars: oscillations (including pulsations)

\section{Introduction}

The title of this review consists of three parts. The adjective asteroseismic is derived from "asteroseismology", which refers to the science in which stellar (aster) oscillations (seismo) are studied (logy) to gain information of stars in general, and about their interior in specific. By matching the observed and theoretically predicted frequency spectrum, severe constraints can be obtained on important parameters like the mass, the internal rotation law (rigid or not), the metallicity and the effects of convection (extent of convective overshooting; size of convective layers). For massive stars, the internal structure globally consists of a convective core and a radiative envelope. Stellar oscillations are described by four numbers: (1) the pulsation frequency $f$ or period $P$ (the timescale of reoccurring patterns), (2) the degree $\ell$ (number of node-lines on the surface), (3) the azimuthal number $m$ (number of node-lines on the surface perpendicular on the equator; the sign is linked to the direction of the propagation) and (4) the radial order $n$ (approximation of the number of node-surfaces between the core and the surface). Different steps in the analysis of the data are needed to determine these numbers: frequency analysis for $f$, mode identification for $\ell$ and $m$ and theoretical modelling for $n$. Based on their surface behaviour, a distinction is made between radial modes $(l=m=0$; only radial motions; symmetry maintained during pulsation cycle) and non-radial modes $(\ell \neq 0$; both radial 
Table 1. Overview of the specifications of the space missions MOST, COROT and KEPLER.

\begin{tabular}{c|c|c|c|}
\hline Space mission & Size telescope & Photometric Accuracy & Length of time-series \\
\hline MOST & $15 / 17.3-\mathrm{cm}$ & few $\mu \mathrm{mag}$ & typically 30 days \\
COROT & $30-\mathrm{cm}$ & $10 \mu \mathrm{mag}$ & $30-150$ days \\
KEPLER & $1.4-\mathrm{m}$ & $1.7 \mu \mathrm{mag}$ & 3.5 years \\
\hline
\end{tabular}

and transverse motions occur; nodelines on surface). In the presence of stellar rotation and/or the presence of a magnetic field, the frequency degeneracy in $m$ is lifted: a nonradial mode with degree $\ell$ will be splitted into its $2 \ell+1$ components, resulting into a so called frequency multiplet. Based on their physical behaviour, a distinction is made between acoustic modes (p-modes, for which the restoring force is pressure) and gravity modes (g-modes, for which the restoring force is buoyancy). For p-modes, the radial motions are dominant (highest amplitudes in core of spectral lines) while for the g-modes the tangential motions are more pronounced (higher amplitudes in the wings of the spectral lines). While p-modes carry information from the surface layers, the g-modes probe the deepest layers of the star, making them very interesting from an asteroseismic point of view. In general, the observed periods for p-modes are shorter then those of g-modes (see below).

At least four different types of observations can be used to assemble the ingredients needed for in-depth asteroseismic studies (a number of well-identified modes and restrictions on stellar parameters), i.e. photometry, spectroscopy, spectropolarimetry and interferometry. Each type has its own advantages and disadvantages. Time-series of photometric observations are in general easy to gather and are particularly useful to detect and study low $\ell$ modes (the amplitude of high- $\ell$ modes are generally too small due to cancellation effects). In the case of multi-colour photometry, the calibration of the photometric system can be used to determine stellar parameters $\left(T_{\text {eff }}, \log g\right.$, metallicity) and reddening, but it only allows a partial mode identification because the observables used in the photometric mode identification technique (photometric amplitude ratios/phase differences, Dupret et al. 2003) only depend on $\ell$ (e.g. Handler et al. 2004; Handler et al. 2006; De Cat et al. 2007). Time-series of spectroscopic observations are complementary to those in photometry in several ways. Low to medium resolution spectra $(\mathrm{R}<30000)$ provide information on extra stellar parameters (radial velocity $v_{\text {rad }}$, projected rotational velocity $v \sin i$, abundances) while time-series of high-quality ( $\mathrm{SNR} \geqslant 200)$, high-resolution $(\mathrm{R} \geqslant 30000)$ spectra are used for a detailed study of line profile variations (LPVs; e.g. De Cat et al. 2005; Briquet et al. 2005; Briquet et al. 2009). They allow a determination of both $\ell$ and $m$ of the observed modes and of constraints on the inclination $i$ and rotational parameters through the application of spectroscopic mode identification techniques (Moment Method: Briquet \& Aerts 2003; Intensity Period Search: Telting \& Schrijvers 1997; Fourier Parameter Fit method: Zima 2006). Moreover, spectroscopic observations are more sensitive to modes with a higher $\ell$ and a lower amplitude because we are not dealing with an integrated quantity. For magnetic stars, spectropolarimetry allows to study magnetic field variations for a determination of the rotation period and the magnetic geometry, which can shrink the free parameter space of the mode identification significantly (Neiner et al. 2003a,b,c) Recently, magnetic field detections have been reported for several OB stars (Hubrig et al. 2006; Hubrig et al. 2007; Hubrig et al. 2009). Also interferometric observations can be useful, in particular for the measurement of the radii of bright nearby stars and for a full orbit determination of binaries (e.g. $\beta$ Cen: Davis et al. 2005; $\lambda$ Sco: Tango et al. 2006). Such observations can either be obtained from observatories on Earth (ground-based) or with space missions (space-based). Because ground-based observations can only be done during the night, time-series of single-site observations are 


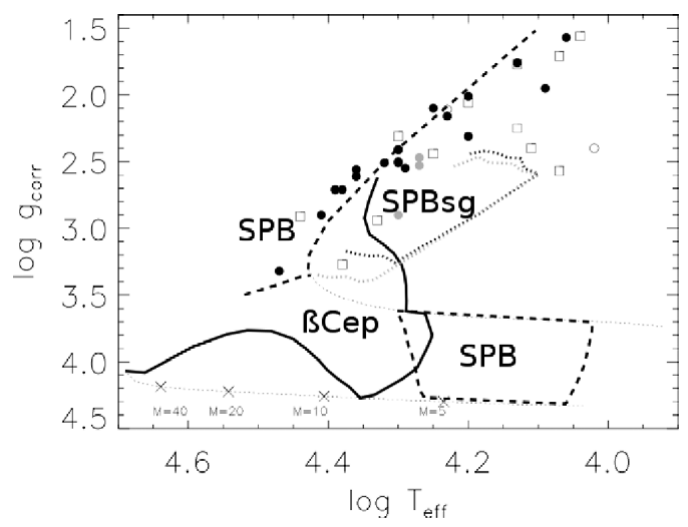

Figure 1. The $\log T_{\text {eff }}-\log g$ diagram for the OB-stars. It shows the theoretical instability strip for low- $n$ p/g-modes (" $\beta$ Cep"; thick solid line) and for high- $n$ g-modes ("SPB"; thick dashed line) calculated by Pamyatnykh (1999), and for g-modes in post-TAMS models for $\ell=1$ (grey dotted line) and $\ell=2$ (black dotted line) as calculated by Saio et al. (2006) ("SPBsg"). The circles and squares indicate the objects studied by Lefever et al. (2007). (altered version of figure taken from Lefever et al. 2007)

generally heavily affected by aliasing effects (spurious frequencies introduced by the time sampling) which hampers the frequency analysis (e.g. Uytterhoeven et al. 2007). These aliasing can partly be solved by performing multi-site campaigns (e.g. $\nu$ Eri: Handler et al. 2004; Aerts et al. 2004a). Asteroseismic space missions like MOST, COROT and KEPLER generally allow continuous photometric monitoring of stars for long periods (see Table 1). Moreover, the quality of the data is not degraded by the Earth's atmosphere which leads to time-series of white-light photometry with a stunning accuracy down to micromagnitude level (Michel et al. 2008; Jenkins et al. 2010; Gilliland et al. 2010) and free from the typical alias frequencies encountered in ground-based observations.

This raises the question: Are space-based observations sufficient for a state-of-theart asteroseismic study? Or, in other words: Are ground-based follow-up observations really worth the effort? In what follows, we will give an answer by focussing on the main asteroseismic results obtained recently for types of variable $O B$ stars, i.e. the hot main-sequence pulsators ( $\beta$ Cephei stars, slowly pulsating B stars and hybrid pulsators; Section 2), the Be stars (Section 3) and OB-supergiants (Section 4).

\section{Hot main-sequence pulsators}

The hot end of the main-sequence hosts two well-established classes of pulsating stars. $\beta$ Cephei $(\beta \mathrm{Cep})$ stars are early B-type (B 0-B 3) main-sequence stars that oscillate in low-radial-order $n \mathrm{p} / \mathrm{g}$-modes for which the observed periods are short (2-12 hours) and the amplitudes low (in general $<40 \mathrm{mmag}$ in light and $<20 \mathrm{~km} \mathrm{~s}^{-1}$ in radial velocity) (see Stankov \& Handler 2005 for a review). Slowly pulsating B (SPB) stars are mid to late B-type (B 2-B 9) main-sequence stars pulsating in high-radial-order $n$ g-modes with longer periods (0.3-5 days) and lower amplitudes (in general $<20 \mathrm{mmag}$ in light and $<10 \mathrm{~km} \mathrm{~s}^{-1}$ in radial velocity) (see De Cat 2007 for a review). The pulsations in both classes of pulsating stars are driven by the $\kappa$ mechanism acting on the iron opacity bump at around 200000 K (e.g. Dziembowski et al. 1993, Gautschy \& Saio 1993). Their theoretical instability strips as derived by Pamyatnykh (1999) are given in Fig. 1. Theoretical frequency spectra of $\beta$ Cep stars are rather sparse making asteroseismic modeling possible with a small number of well-identified modes. For SPB stars, much more well-identified modes are needed because their theoretical frequency spectra are much denser. 
Table 2. Overview of the results of in-depth asteroseismic results obtained for $\beta$ Cep stars so far. In the colums, we give for each object the HD number, the variable star name (if available), the spectral type, the numbers of modes used for the asteroseismic modeling, the identification of the radial mode, the overshoot, the velocity of the rotation core, the detection of g-modes, and the references, respectively.

\begin{tabular}{|c|c|c|c|c|c|c|c|c|}
\hline HD number & Name & SpT & \# fit & radial mode & $\alpha_{\mathrm{ov}}$ & rotation core & $\mathrm{g}$ & Ref \\
\hline HD 129929 & V836 Cen & B3V & 3 & fundamental & $0.10(5)$ & urf. & no & {$[1],[2]$} \\
\hline HD 29248 & $\nu$ Eri & B2III & 4 & fundamental & $0.05(5)$ & $3 \mathrm{x}$ faster than surf. & yes & {$[3],[4]$} \\
\hline HD 16582 & $\delta$ Ceti & B2IV & 2 & 1st overtone & $0.20(5)$ & & no & {$[5]$} \\
\hline HD 44743 & $\beta \mathrm{CMa}$ & B2III & 2 & 1st overtone & $0.20(5)$ & & no & {$[6]$} \\
\hline HD 157056 & $\theta \mathrm{Oph}$ & B2IV & 3 & fundamental & $0.44(7)$ & (not conclusive) & no & {$[7]$} \\
\hline HD 214993 & $12 \mathrm{Lac}$ & B2III & $2+$ & 1st overtone & $<0.4$ & (faster than surf.?) & yes & {$[8]$} \\
\hline
\end{tabular}

[1] Aerts et al. (2003), [2] Dupret et al. (2003), [3] Ausseloos et al. (2004), [4] Pamyatnykh et al. (2004), [5] Aerts et al. (2006b), [6] Mazumdar et al. (2006), [7] Briquet et al. (2007), [8] Desmet et al. (2009)

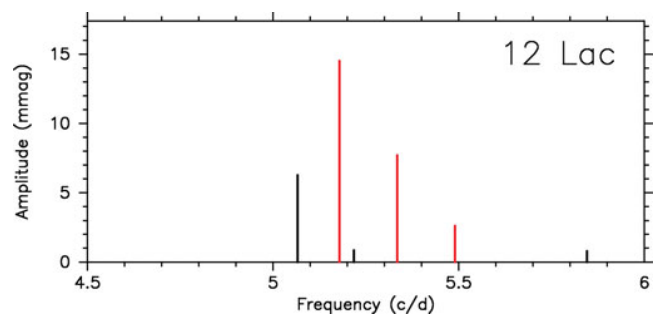

Figure 2. The observed frequencies of the $\beta$ Cep star 12 Lac. The frequencies indicated in red (colours in electronic version only) give the false impression that we are dealing with a rotationally splitted $\ell=1$ mode.

The first in-depth asteroseismic study of a $\beta$ Cephei ( $\beta$ Cep) star was solely based on single-site ground-based photometry. In a time-span of 21 years (!), a total of about 1500 observations in the 7-colour photometric system of Geneva were collected for V836 Cen (HD 129929), resulting in the detection of six frequencies (Aerts et al. 2004b). These were identified with the radial fundamental $\left(\ell=0, \mathrm{p}_{1}\right)$ mode, two components of the $\ell=1, \mathrm{p}_{1}$ mode and two components of the $\ell=2, \mathrm{~g}_{1}$ mode (Dupret et al. 2004). This study gave the first evidence for convective overshooting at the core and, thanks to the observations of two rotationally splitted modes, of non-rigid rotation (Aerts et al. 2003).

The best studied $\beta$ Cep star solely based on ground-based observations is $\nu$ Eri, for which a large scale photometric and spectroscopic multi-site campaign was organised in 2002-2003, with an extension of the photometric multi-site campaign to 2004. The frequency analysis of the data revealed 34 frequencies in photometry (14 independent and 20 combination frequencies; Handler et al. 2004; Jerzykiewicz et al. 2005) and 20 frequencies in spectroscopy ( 8 independent and 12 combination frequencies; Aerts et al. 2004a; De Ridder et al. 2004), including two g-modes, making $\nu$ Eri a so-called $\beta$ Cep/SPB hybrid pulsator (see below). For the modeling, the frequencies of the four identified modes can be used: $\ell=0, \mathrm{p}_{1}, \ell=1, \mathrm{~g}_{1}, \ell=1, \mathrm{p}_{1}$, and $\ell=1, \mathrm{p}_{2}$. By doing so, four of the five free parameters $\left(X, Z, \alpha_{\mathrm{ov}}, M\right.$, age) can be easily determined with standard models, but a problem with mode excitation occurs: the $\ell=1, \mathrm{p}_{2}$ is not excited in the resulting model. Different solutions have been proposed to solve this problem. Ausseloos et al. (2004) assumed a global metal enhancement while Pamyatnykh et al. (2004) suggested a local iron enrichment in the driving zone due to diffusion.

In Table 2 we give an overview of the in-depth asteroseismic studies of $\beta$ Cep stars, from the era before the COROT satellite, enforcing the main conclusions of the studies 


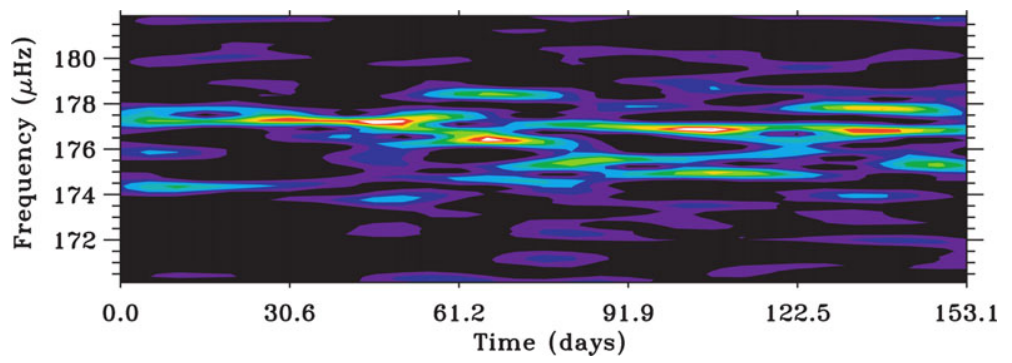

Figure 3. Time-frequency diagram, using a Morlet wavelet with a 20-day width, showing the solar-like oscillations of V1449 Aql after prewhitening the $\beta$ Cep and SPB modes. (figure taken from Belkacem et al. 2009).

highlighted above. Fig. 2 shows the observed photometric frequencies of 12 Lac (Handler et al. 2006). From the nearly equidistant frequencies given in red, one might conclude that 12 Lac exhibits a rotationally split $\ell=1$ mode and use this information for asteroseismic modeling. However, spectroscopic mode identification revealed that these frequencies originate from an $(\ell, m)=(1,1)$ mode (left), a radial mode (middle) and an $(2,1)$ mode (right) (Desmet et al. 2009). This example shows that asteroseismic modeling without mode identification should be avoided, and illustrates the need for multi-colour and spectroscopic information in seismic studies of B-type stars. Indeed, space missions, such as COROT and KEPLER only provide white-light photometry, which does not allow direct mode-identification. The optimal scientific exploitation of the seismic space data requires complementary ground-based data (e.g. Uytterhoeven 2009; Uytterhoeven et al. 2010a,b).

However, COROT has lead to important new results for $\beta$ Cep stars as can be illustrated by the stories of V1449 Aql (HD 180642) and HD 46149. Before COROT, the B1.5II-III star V1449 Aql was known as a monoperiodic $\beta$ Cep star with a radial mode $\left(5.4871 d^{-1}\right)$ of very high amplitude showing strong non-linear effects (Aerts 2000). It was observed for 156 days by COROT and an intensive ground-based follow-up campaign in multi-colour photometry and high-resolution spectroscopy was organised. The new datasets show that V1449 Aql is clearly multiperiodic and exhibits both $\beta$ Cep and SPB-like frequencies. Two of the newly found frequencies are identified with $\ell=3$ modes (Briquet et al. 2009). The 11 independent and 22 low-order combination frequencies discovered in the COROT data were modeled in detail by comparing three different models of which nonlinear resonant mode coupling seems to be the statistically preferred one (Degroote et al. 2009b). Moreover, V1449 Aql is the first massive star for which hints for damped acoustic modes excited by turbulent convection associated with the Fe-opacity bump in the upper layers are found (Fig. 3; Belkacem et al. 2009). Evidence for solar-like oscillations for which the observed frequency range and spacings are compatible with theoretical predictions is also found for the late O-type star HD 46149 based on observations of the COROT satellite (Degroote et al. 2010b).

Compared to these successes, asteroseismic studies of slowly pulsating B (SPB) stars are still in their infancy. This is mainly caused by observational constraints: typical pulsation periods are of the order of a day, what makes it extremely difficult to monitor the periodic variations from the ground, and amplitudes are fairly small (below 20 mmag; $10 \mathrm{~km} \mathrm{~s}^{-1}$ ). Moreover, the abovementioned techniques currently used for spectroscopic mode identification have been developed and extensively tested for p-mode pulsations. While these methods are often successful for p-mode pulsators, their application to g-modes seems not to be straightforward (De Cat et al. 2005; Zima et al. 2007). 

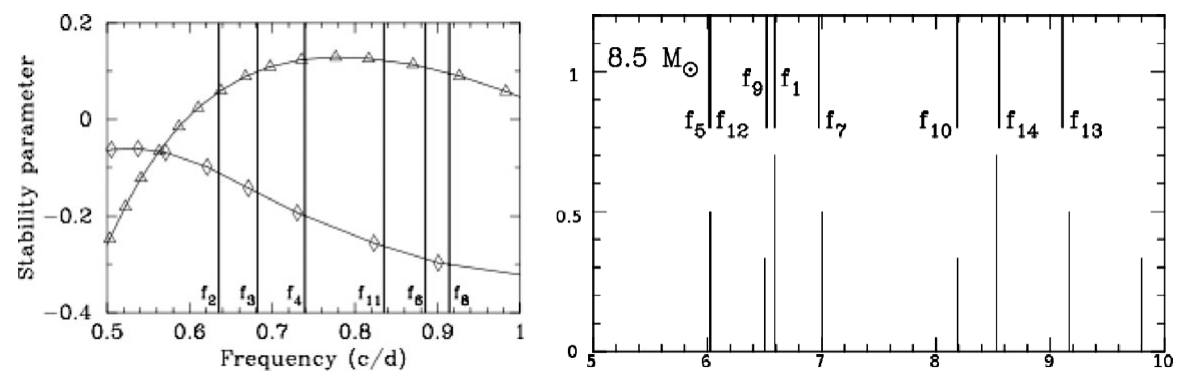

Figure 4. Comparison of theoretically predicted pulsation frequencies of an $8.5 \mathrm{M}_{\odot}$ model of $\gamma$ Peg fitting $f_{1}(\ell=0)$ and $f_{5}(\ell=1)$ with the observed pulsation frequencies indicated with $f_{1}, \ldots, f_{14}$. Left: Stability of the theoretical $\ell=1$ (diamonds) and $\ell=2$ (triangles) modes. If the stability parameter is above (below) zero, the corresponding mode is excited (stable). Right: The model frequencies are plotted at the bottom with arbitrary amplitudes of 0.7 for $\ell=0,0.5$ for $\ell=1$, and 0.33 for $\ell=2$. (figures taken from Belkacem et al. 2009).

Consequently, not many well-studied cases exist. Dedicated ground-based spectroscopic multi-site campaigns for main-sequence g-mode pulsators are currently ongoing in an attempt to fill this void (De Cat et al. 2009).

Since the discovery of SPB stars by Waelkens (1991), HD 160124 has remained the record holder for the maximum number of frequencies for 15 years ( 8 frequencies between 0.3 and $1.1 d^{-1}$ ). Thanks to 37 days of continuous monitoring with the satellite MOST, up to 20 significant frequencies were detected in the resulting time-series of whitelight photometry of the B5 II/III star HD 163830 (Aerts et al. 2006a). The two lowest frequencies were tentatively attributed to stellar rotation while the remaining 18 frequencies are consistent with low- $\ell$, high- $n$ nonradial g-modes of seismic models of an evolved $4.5 \mathrm{M}_{\odot}$ star. This study nicely illustrates that space-based data are able to increase the number of observed pulsation frequencies significantly (see also Poretti et al. 2009; Chapellier et al. 2010), but the lack of a formal mode identification prevents us to move on to asteroseismic modeling.

The theoretical instability strips of $\beta$ Cep and SPB stars have a common part that should host stars in which low- $n \mathrm{p} / \mathrm{g}$-modes and high- $n$ g-modes are simultaneously excited (Fig. 1). The existence of the so called hybrid pulsators was already illustrated above. They are theoretically predicted (e.g. Miglio et al. 2007) and are of particular asteroseismic interest because different internal regions can be probed at the same time.

$\gamma$ Peg (HD 886) was one of the first suspected hybrid $\beta$ Cep/SPB stars (Chapellier et al. 2006). It was recently subjected to a detailed study based on 10 weeks of radial velocity monitoring and 30 days of white-light photometry from the MOST satellite, which lead to the detection of eight $\beta$ Cep-modes and six SPB-modes (Handler et al. 2009). The observed p-modes of $\gamma$ Peg can be fitted extremely well with an $8.5 \mathrm{M}_{\odot}$ model (right panel of Fig. 4). Note that all the expected p-modes are observed! Only a minor frequency difference is found between $f_{13}$ and its theoretical counterpart, i.e. an $\ell=1, \mathrm{p}_{2}$ mode $(=$ the p-mode for which the excitation problems were encountered in the analysis of $\nu$ Eri; see above). The low $v \sin i$ value of $\gamma \operatorname{Peg}\left(\sim 3 \mathrm{~km} \mathrm{~s}^{-1}\right)$ is consistent with the assumption that $f_{5}$ and $f_{12}$ are components of a rotationally splitted $\ell=1$ mode. Also the frequency spacings of the observed g-modes agree well with those of the theoretically predicted $\ell=1$ modes, but these dipole modes are all stable (left panel of Fig. 4). The systematic shift to lower frequencies might be indicative that all the observed g-modes are prograde dipole modes, which seems to be common amongst main-sequence g-mode pulsators (Wright et al., in preparation). As the authors say themselves, this study is "a demonstration of the value of combining space based photometry with ground-based spectroscopy". 


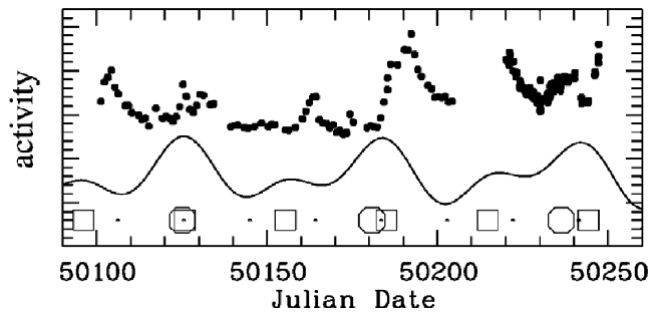

Figure 5. Circumstellar activity (points) of $\mu$ Cen in 1996 compared to the combined amplitude of the multiperidicity (solid line). The symbols mark times when two strong modes have no phase difference. (figure taken from Rivinius et al. 1998c).

The most exciting hybrid discovery was done for the young B3V star HD 50230, a star that has been observed for 137 days with the COROT satellite. The data revealed its hybrid nature as hundreds of g-modes with mmag amplitudes and tens of p-modes with $\mu$ mag amplitudes were detected. More importantly, there is strong evidence for a quasi uniform period spacing in the observed g-modes (Degroote et al. 2010a). Such a period spacing is predicted for high- $n$ g-modes in the asymptotic regime but was never observed before. The deviations from a uniform period spacing could be nicely fitted with a sinusoid with a period of $2450 \mathrm{sec}$ and a decreasing amplitude with a maximum of 240 sec. These results imply that already $60 \%$ of the initial hydrogen is consumed and that there is a smooth gradient of chemical composition outside the convective core.

The examination of the light curves of 358 candidate B pulsators observed in the exoplanet field of the initial run of COROT ( 55 days) has lead to a wealth of objects with different types and flavours (Degroote et al. 2009a). A subset of these variable stars fills the gap between the theoretical instability strip of the SPB and the $\delta$ Scuti stars but their true nature can only be unraveled if (preferably high-resoltion) spectra would become available (Uytterhoeven 2009; cf. ongoing large program on the ESO/Flames multi-fiber spectrograph; P.I. C. Neiner). There is a similar need for classification spectra for the candidate B-type pulsators that are currently being observered with the space mission KEPLER (Uytterhoeven et al. 2010a,b; Lehmann et al. 2010).

\section{Be stars}

Be stars are non-supergiant B-type stars for which emission lines are observed at least once (see Porter \& Rivinius 2003 for a review). The objects are flattened because of their very fast rotation. Be stars show long term variations that are linked to episodes of mass ejections (outbursts), leading to a decretion disk. It is still not clear what causes these outbursts. Be stars also show short term variations that could be a reflection of stellar pulsations ( $\beta$ Cep-type modes, SPB-type modes) and/or of rotational modulation (spots, clouds). So the key question for Be star research is: What causes the Be phenomenon?

The B2IV-Ve star $\mu$ Cen (HD 120324) has been observed intensively with ground-based instruments in the nineties (Rivinius et al. 1998a,b; Rivinius et al. 2001). A total of six frequencies were found that seemed to be phase coherent over five years. By comparing the circumstellar activity with the combined amplitude of the multiperiodic variations, there is clear evidence that the times of the combined maxima coincide with the times of the onset of the outbursts (Fig. 5; Rivinius et al. 1998c). This seems to suggest that pulsation triggers the mass transfer from the star to the disk.

The satellite MOST has provided data for several studies of massive emission-line stars. The 24 days of MOST observations of the O9.5Ve star $\zeta$ Oph (HD 149757) have revealed 16 significant frequencies, of which six are in common with the eight significant frequencies 
Table 3. Overview of the SPBe stars that have been observed by the MOST satellite. In the colums, we give for each object the HD number, the variable star name (if available), the spectral type, the total time base of the MOST observations (in days), the rotation frequency (in $d^{-1}$ ), the number of detected frequencies, the number of frequency groups, and the references, respectively.

\begin{tabular}{|c|c|c|c|c|c|c|c|c|}
\hline HD number & Name & SpT & MOst & \multicolumn{2}{|c|}{$f_{\text {rot }}$} & \#freq & \#groups $\mid$ Ref \\
\hline HD 127756 & & B1/B2Vne & 31 d & 0.86 & $d^{-1}$ & 30 & 3 & {$[1]$} \\
HD 163868 & & B5Ve & 37 d & 1.38 & $d^{-1}$ & 60 & 3 & {$[2]$} \\
HD 217543 & & B3Vpe & 26 d & 1.73 & $d^{-1}$ & 40 & 3 & {$[1]$} \\
HD 58715 & $\beta$ CMi & B8Ve & 41 d & 2.76 & $d^{-1}$ & 20 & 1 & {$[3]$} \\
\hline
\end{tabular}

[1] Cameron et al. (2008), [2] Walker et al. (2005b), [3] Saio et al. (2007).

detected in the varations of ground-based high-resolution spectra (Walker et al. 2005a). The dominant mode with frequency $5.1806 d^{-1}$ is interpreted as the radial first overtone. The observed frequencies are compatible with low- $n$ radial and nonradial $\mathrm{p} / \mathrm{g}$-modes modified by rotation, i.e. $\beta$ Cep-pulsations excited by the $\kappa$ mechanism. Therefore, we could call $\zeta$ Oph a $\beta$ Cephei Be ( $\beta$ Cepe) star. Unfortunately, no unambiguous rotational frequency could be identified for $\zeta$ Oph.

For HD 163868, up to 60 significant frequencies are detected in the MOsT lightcurve spanning 37 days (Walker et al. 2005b). The observed groups around 14 and 7 hours are compatible with prograde $|m|=1$ and $|m|=2$ even g-modes, respectively ( $\ell-m$ is even (odd) for "even (odd) modes"). The tail of the observed group around 8 days coincides with a group of unstable retrograde $|m|=1$ odd r-modes (Rossby modes) predicted by representative seismic models. Hence, the observed frequencies can be interpreted as nonradial $\mathrm{g} / \mathrm{r}$-modes distorted by fast rotation and excited by the $\kappa$ mechanism, making HD 163868 the prototype of the slowly pulsating Be (SPBe) stars. Three other SPBe stars have been detected thanks to the MOST satellite (Saio et al. 2007; Cameron et al. 2008). A short overview of their results is given in Table 3. Cameron et al. (2008) derived from the resulting rotation frequencies that all these SPBe stars are rotating close to the critical value. Their rotation frequency decreases with increasing temperature which is a reflection the increase in radius.

The satellite COROT has made an important contribution in Be star research. In Table 4, a short summary of the results for the observed stars is given (for details: see overview of Gutiérrez-Soto et al., these proceedings). HD 49330 showed a moderate outburst of $30 \mathrm{mmag}$ during the COROT observations (Huat et al. 2009; Floquet et al. 2009). The outburst could be subdivided in four phases: quiescent, precursor, outburst and relaxation. The amplitudes of the observed $\mathrm{g}(\mathrm{p})$-modes in(de)creased during the precursor and outburst phase, while they de(in)creased during the relaxation phase. Like for $\mu$ Cen, this indicates that the pulsations might cause the outburst. Or is it the outburst that causes the excitation of pulsation modes - a possible scenario suggested by Owocki (2005)? The COROT data of HD 50209 has provided proof that g-mode pulsations occur in late Be stars (Diago et al. 2009). Indeed, the rotational frequency $f_{\text {rot }}=0.679 d^{-1}$ has been observed together with a rotationally split g-mode with $m=0,-1,-2,-3\left(0.10811 d^{-1}+\mathrm{n} f_{\text {rot }}\right.$ with $\mathrm{n}=0,1,2,3)$. The observed variations of HD 175869 can be fully explained by rotational modulation, but nonradial pulsations can not be excluded (Gutiérrez-Soto et al. 2009). If this star exhibits a dipolar magnetic field, then the longitudinal component must be below 400 Gauss. For HD 181231, the frequency $0.624 d^{-1}$ is compatible with the rotation frequency (as derived from the fundamental parameters) and $0.695 d^{-1}$ is interpreted as a nonradial g-mode with $\ell \sim 3$ (Neiner et al. 2009). The observed 14-day modulation is due to beating between $f_{\text {rot }}$ and the g-mode, while an additional modulation with a period of 
Table 4. Overview of the Be stars that have been observed by the COROT satellite. In the columns, we give for each object the HD number, the spectral type, the total time base of the COROT observations (in days), the rotation frequency (in $d^{-1}$ ), the detection of g-modes, the detection of p-modes, the variability of the photometric amplitudes of the modes, the variability of the spectral lines, the detection of a magnetic field, and the references, respectively.

\begin{tabular}{|c|c|c|c|c|c|c|c|c|c|}
\hline HD number & SpT & COROT & $f_{\text {rot }}$ & $\mathbf{g}$ & $\mathbf{p}$ & amplitude & LPVs & $\bar{B} \mid$ Ref \\
\hline HD 49330 & B0.5IVe & 136 d (LRa1) & $0.87 d^{-1}$ & yes & yes & yes & yes & $\mid[1],[2]$ \\
HD 50209 & B8IVe & 136 d (LRa1) & $0.679 d^{-1}$ & yes & no & yes & no & & {$[3]$} \\
HD 175869 & B8IIIe & 27.3 d (SRc1) & $0.639 d^{-1}$ & (no) & (no) & yes & no & no & {$[4]$} \\
HD 181231 & B5IVe & 157 d (LRc1) & $0.624 d^{-1}$ & yes & no & yes & yes & no & {$[5]$} \\
\hline
\end{tabular}

[1] Huat et al. (2009), [2] Floquet et al. (2009), [3] Diago et al. (2009), [4] Gutiérrez-Soto et al. (2009), [5] Neiner et al. (2009).

116 days can be due either to beating or a zonal mode. This star can not host a dipolar magnetic field with a longitudinal component above 650 Gauss. It is clear that pulsations are present in early, mid and late Be stars, indicating that Be stars have an excellent asteroseismic potential. No LPVs were detected for HD 50209 and HD 175869 while the periods present in the variations of the photospheric lines of HD 49330 and HD 181231 could be linked with those of the photometry. For the latter stars, also variations in the emission lines were found which means that their disk is either being generated or is changing/moving. Note that complementary ground-based follow-up observations were needed for the detection of the rotation frequency, for the study of the variability of the spectral lines, and for the search for magnetic fields!

\section{OB-Supergiants}

OB-Supergiants are massive, evolved stars. The presence of non-radial pulsations in OB-supergiants was first suggested in the context of an explanation for the semi-regular light and radial variations observed in $\alpha$ Cygni variables (periods of 5 - 100 days; amplitudes of 0.01 - 0.1 mmag; e.g. van Genderen 2001).

Recently, more firm evidence for non-radial pulsations in a B supergiant was found, thanks to 37 days of observations with the satellite MOsT. A total of 48 frequencies were detected for the B2Ib/II star HD 163899 (Saio et al. 2006). These frequencies range from 0 to $3 d^{-1}$, embracing both g- and p-mode pulsations. Indeed, according to the seismic models for post-TAMS stars of Saio et al. (2006), both p- and g-modes can be unstable in OB-supergiants (Fig. 6). These g-modes have a great asteroseismic potential and can be excited because they are reflected at the convective zone associated to the hydrogen-burning shell. The location of HD 163899 in the H-R diagram is compatible with the picture of simultaneous p- and g-modes (Fig. 6). This star is considered as the first member of the slowly pulsating B supergiant (SPBsg) stars, which are distinct from the $\alpha$ Cygni variables. The theoretical instability strips for $\ell=1$ and $\ell=2$ g-modes in SPBsg stars based on the models of Saio et al. (2006) are given in Fig. 1.

More observational evidence for g-mode instabilities in OB-supergiants was found by Lefever et al. (2007). They carefully derived the atmospheric and wind parameters of a sample of 28 periodically variable and 12 comparison B supergiants. These variable B supergiants were classified as such by Waelkens et al. (1998) and show variations with periods ranging from 1 to 25 days. Several of them show evidence for multi-periodicity. Their resulting location in the H-R diagram is consistent with the suggestion that the observed variations are due to opacity driven g-modes (circles and squares in Fig. 1).

More evidence for g-mode pulsations is found from an unexpected angle. For OBsupergiants, the total line broadening of spectral lines can only be explained after 


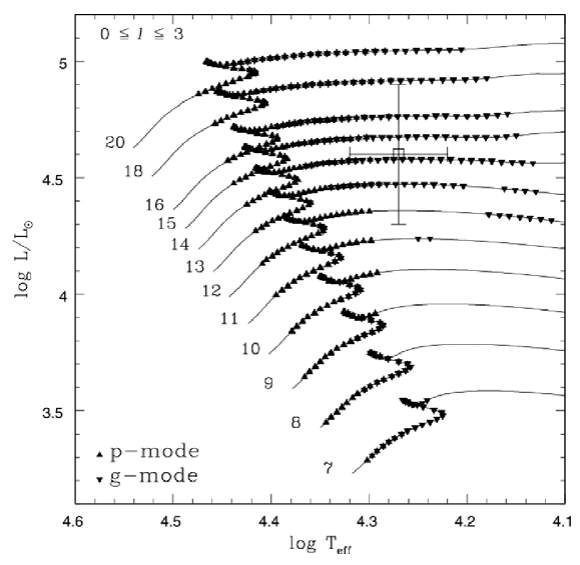

Figure 6. On the evolutionary tracks of massive stars, the seismic models with unstable p-modes are indicated with triangles and those with unstable g-modes with inverted triangles (only modes up to $\ell=3$ are considered). The square with error bars indicates the estimated position of HD 163899 which is compatible with the picture of the simultaneous existence of p- and g-modes. (figure taken from Saio et al. 2006).

introduction of an important extra line-broadening called macroturbulence. It was believed to originate from large-scale turbulent motions but this interpretation has recently been questioned because it would imply highly supersonic velocity fields in many cases. Aerts et al. (2009) suggested the occurrence of hundreds of low-amplitude non-radial g-modes as an alternative physical explanation for macroturbulence. Simón-Díaz et al. (2010) examined spectroscopic observations for a sample of 12 O9.5-B2 supergiant stars. They found firm observational evidence for a strong correlation between the average size of the macroturbulence and the peak-to-peak amplitudes in the first (centroid velocity) and third (skewness) normalised velocity moment of the photospheric LPVs. These observed observations agree extremely well with those predicted by the simulations of Aerts et al. (2009), making g-mode pulsations a valid explanation for macrotubulence. A dedicated ground-based spectroscopic follow-up campaign for selected OB-supergiants is currently ongoing (Simón-Díaz et al. 2009), promising new exciting prospects for the seismic analysis of the not well-studied class of OB-supergiants.

The luminous blue variable supergiant HD 50064 has been observed for 137 days by the satellite COROT. Afterwards, 14 ground-based high-resolution échelle spectra were gathered at three epochs in a time span of 169 days by Aerts et al. (2010). They detected a period of 37 days in the time series of COROT photometry and discovered a sudden amplitude increase with a factor of 1.6 that occurred once. The photometric period was also observed in the spectroscopic variations. The mass loss rate, as derived from the Balmer lines, seems to undergo variations on a similar timescale. The observed period is tentatively interpreted as a radial strange mode oscillation that is triggering periodic mass-loss episodes in which a circumstellar envelope is being generated.

\section{Conclusions}

A better knowledge of the internal structure and the evolution of massive stars is of great importance because they are the progenitors of type II, Ib or Ic supernovae. These stars enrich their immediate environment with heavy elements at the end of their lives. We have discussed the results of some representative examples to prove that the $\beta$ Cephei, slowly pulsating B, Be and OB-supergiant stars all have an excellent asteroseismic potential. Recently, progress has already been made thanks to the space missions MOST and 
COROT, and even more exciting results are being expected in the near future from the KEPLER mission too. However, as we have shown, complementary ground-based support has been essential to get these results and will remain mandatory to make even more progress in the future!

\section{References}

Aerts, C. 2000, $A \mathscr{E} A, 361,245$

Aerts, C., De Cat, P., Handler, G., \& Heiter, U. et al. 2004a, MNRAS, 347, 463

Aerts, C., De Cat, P., Kuschnig, R., \& Matthews, J. M. et al. 2006a, ApJ (Letters), 642, L165

Aerts, C., Lefever, K., Baglin, A., \& Degroote, P. et al. 2010, A\&A, 513, L11

Aerts, C., Marchenko, S. V., Matthews, J. M., \& Kuschnig, R. et al. 2006b, ApJ, 642, 470

Aerts, C., Puls, J., Godart, M., \& Dupret, M.-A. 2009, A\& A, 508, 409

Aerts, C., Thoul, A., Daszyńska, J., \& Scuflaire, R. et al. 2003, Science, 300, 1926

Aerts, C., Waelkens, C., Daszyńska-Daszkiewicz, J., \& Dupret, M.-A. et al. 2004b, A\&A, 415, 241

Ausseloos, M., Scuflaire, R., Thoul, A., \& Aerts, C. 2004, MNRAS, 355, 352

Belkacem, K., Samadi, R., Goupil, M.-J., \& Lefèvre, L. et al. 2009, Science, 324, 1540

Briquet, M. \& Aerts, C. 2003, A\& A, 398, 687

Briquet, M., Lefever, K., Uytterhoeven, K., \& Aerts, C. 2005, MNRAS, 362, 619

Briquet, M., Morel, T., Thoul, A., \& Scuflaire, R. et al. 2007, MNRAS, 381, 1482

Briquet, M., Uytterhoeven, K., Morel, T., \& Aerts, C. et al. 2009, A\&GA, 506, 269

Cameron, C., Saio, H., Kuschnig, R., \& Walker, G. A. H. et al. 2008, ApJ, 685, 489

Chapellier, E., Le Contel, D., Le Contel, J. M., \& Mathias, P. et al. 2006, A\& A, 448, 697

Chapellier, E., Rodríguez, E., Auvergne, M., Uytterhoeven K., et al. 2010, A\&A, 525, A23

Davis, J., Mendez, A., Seneta, E. B., \& Tango, W. J. et al. 2005, MNRAS, 356, 1362

De Cat, P. 2007, Communications in Asteroseismology 150, 167

De Cat, P., Briquet, M., Aerts, C., \& Goossens, K. et al. 2007, A\& A, 463, 243

De Cat, P., Briquet, M., Daszyńska-Daszkiewicz, J., \& Dupret, M. A. et al. 2005, A $\& A$, 432, 1013

De Cat, P., Wright, D. J., Pollard, K. R., \& Maisonneuve, F. et al. 2009, in: J. A. Guzik \& P. A. Bradley (eds.), American Institute of Physics Conference Series, AIP-CP 1170, p. 480

Degroote, P., Aerts, C., Baglin, A., \& Miglio, A. et al. 2010a, Nature, 464, 259

Degroote, P., Aerts, C., Ollivier, M., \& Miglio, A. et al. 2009a, A\&A, 506, 471

Degroote, P., Briquet, M., Auvergne, M., \& Simón-Díaz, S. et al. 2010b, A\&A, 519, A38

Degroote, P., Briquet, M., Catala, C., \& Uytterhoeven, K. et al. 2009b, AESA, 506, 111

De Ridder, J., Telting, J. H., Balona, L. A., \& Handler, G. et al. 2004, MNRAS, 351, 324

Desmet, M., Briquet, M., Thoul, A., \& Zima, W. et al. 2009, MNRAS, 396, 1460

Diago, P. D., Gutiérrez-Soto, J., Auvergne, M., \& Fabregat, J. et al. 2009, A\&̈A, 506, 125

Dupret, M.-A., De Ridder, J., De Cat, P., \& Aerts, C. et al. 2003, A\&A, 398, 677

Dupret, M.-A., Thoul, A., Scuflaire, R., \& Daszyńska-Daszkiewicz, J. et al. 2004, A\&3A, 415, 251

Dziembowski, W. A., Moskalik, P., \& Pamyatnykh, A. A. 1993, MNRAS, 265, 588

Floquet, M., Hubert, A.-M., Huat, A.-L., \& Frémat, Y. et al. 2009, A\&̈A, 506, 103

Gautschy, A. \& Saio, H. 1993, MNRAS, 262, 213

Gilliland, R. L., Jenkins, J. M., Borucki, W. J., \& Bryson, S. T. et al. 2010, ApJ (Letters), 713, L160

Gutiérrez-Soto, J., Floquet, M., Samadi, R., \& Neiner, C. et al. 2009, A\&A, 506, 133

Handler, G., Jerzykiewicz, M., Rodríguez, E., \& Uytterhoeven, K. et al. 2006, MNRAS, 365, 327

Handler, G., Matthews, J. M., Eaton, J. A., \& Daszyńska-Daszkiewicz, J. et al. 2009, ApJ (Letters), 698, L56 
Handler, G., Shobbrook, R. R., Jerzykiewicz, M., \& Krisciunas, K. et al. 2004, MNRAS, 347, 454

Huat, A.-L., Hubert, A.-M., Baudin, F., \& Floquet, M. et al. 2009, A\&A, 506, 95

Hubrig, S., Briquet, M., De Cat, P., \& Schöller, M. et al. 2009, AN, 330, 317

Hubrig, S., Briquet, M., Schöller, M., \& De Cat, P. et al. 2006, MNRAS, 369, L61

Hubrig, S., Briquet, M., Schöller, M., \& De Cat, P. et al. 2007, in: A. T. Okazaki, S. P. Owocki, \& S. Stefl (eds.), Active OB-Stars: Laboratories for Stellar and Circumstellar Physics, ASPCS 361, p. 434

Jenkins, J. M., Caldwell, D. A., Chandrasekaran, H., \& Twicken, J. D. et al. 2010, ApJ (Letters), 713, L120

Jerzykiewicz, M., Handler, G., Shobbrook, R. R., \& Pigulski, A. et al. 2005, MNRAS, 360, 619

Lehmann, H, Tkachenko, A., Semaan, T., Gutiérrez-Soto, J. et al. 2010, A\&\&A, 526, A124

Lefever, K., Puls, J., \& Aerts, C. 2007, A\& A, 463, 1093

Mazumdar, A., Briquet, M., Desmet, M., \& Aerts, C. 2006, A\&A A, 459, 589

Michel, E., Baglin, A., Auvergne, M., \& Catala, C. et al. 2008, Science, 322, 558

Miglio, A., Montalbán, J., \& Dupret, M.-A. 2007, MNRAS, 375, L21

Neiner, C., Geers, V. C., Henrichs, H. F., \& Floquet, M. et al. 2003a, A\&SA, 406, 1019

Neiner, C., Gutiérrez-Soto, J., Baudin, F., \& de Batz, B. et al. 2009, A\&A, 506, 143

Neiner, C., Henrichs, H. F., Floquet, M., \& Frémat, Y. et al. 2003c, $A \& A, 411,565$

Neiner, C., Hubert, A.-M., Frémat, Y., \& Floquet, M. et al. 2003b, A\&A A, 409, 275

Owocki, S. 2005, in: R. Ignace \& K. G. Gayley (eds.), The Nature and Evolution of Disks Around Hot Stars, ASP-CS 337, p. 101

Pamyatnykh, A. A. 1999, AcA, 49, 119

Pamyatnykh, A. A., Handler, G. \& Dziembowski, W. A. 2004, MNRAS, 350, 1022

Poretti, E., Michel, E., Garrido, R., \& Lefèvre, L. et al. 2009, A\&GA, 506, 85

Porter, J. M. \& Rivinius, T. 2003, PASP 115, 1153

Rivinius, T., Baade, D., Stefl, S., \& Stahl, O. et al. 1998a, A\&A, 333, 125

Rivinius, T., Baade, D., Stefl, S., \& Stahl, O. et al. 1998b, A\&A, 336, 177

Rivinius, T., Baade, D., Stefl, S., \& et al. 1998c, in: L. Kaper \& A. W. Fullerton (eds.), Cyclical Variability in Stellar Winds, p. 207

Rivinius, T., Baade, D., Štefl, S., \& Townsend, R. H. D. et al. 2001, A\& $A, 369,1058$

Saio, H., Cameron, C., Kuschnig, R., \& Walker, G. A. H. et al. 2007, ApJ, 654, 544

Saio, H., Kuschnig, R., Gautschy, A., \& Cameron, C. et al. 2006, ApJ, 650, 1111

Simón-Díaz, S., Herrero, A., Uytterhoeven, K., \& Castro, N. et al. 2010, ApJ (Letters), 720, L174

Simón-Díaz, S., Uytterhoeven, K., Herrero, A., \& Castro, N. 2009, in: J.A. Guzik \& P.A. Bradley (eds.), Stellar pulsation: Challenges for theory and observation, AIP Conf. Ser., 1170, p. 397

Stankov, A. \& Handler, G. 2005, ApJS, 158, 193

Tango, W. J., Davis, J., Ireland, M. J., \& Aerts, C. et al. 2006, MNRAS, 370, 884

Telting, J. H. \& Schrijvers, C. 1997, A\& A, 317, 723

Uytterhoeven, K. 2009, Communications in Asteroseismology 158, 156

Uytterhoeven, K., Briquet M., Bruntt H., \& De Cat, P. et al. 2010a, AN, in press, arXiv1003.6093

Uytterhoeven, K., Poretti, E., Rodríguez, E., \& De Cat, P. et al. 2007, A\&SA, 470, 1051

Uytterhoeven, K., Szabo, R., Southworth, J., \& Randall, S. et al. 2010b, AN, in press, arXiv1003.6089

van Genderen, A. M. 2001, A\&A, 366, 508

Waelkens, C. 1991, $A \& A$ A, 246, 453

Waelkens, C., Aerts, C., Kestens, E., \& Grenon, M. et al. 1998, A\&A, 330, 215

Walker, G. A. H., Kuschnig, R., Matthews, J. M., \& Reegen, P. et al. 2005a, ApJ (Letters), 623, L145

Walker, G. A. H., Kuschnig, R., Matthews, J. M., \& Cameron, C. et al. 2005b, ApJ (Letters), $635, \mathrm{~L} 77$

Zima, W. 2006, A\&BA, 455, 227

Zima, W., De Cat, P. \& Aerts, C. 2007, Communications in Asteroseismology 150, 189 